\title{
analysis
}

\section{Virtual rice}

\section{Japan sets up the Rice Simulator Project to create an in silico rice plant}

W across the Northern Hemisphere set out to plant a new crop in their paddy fields. At the same time, Japanese researchers have been sowing the seeds for an in silico rice plant that is expected to fertilise research on rice as well as the whole field of plant genomics for years to come. Building on the two draft sequences of the rice genome, published recently in Science, the Japanese government has provided considerable funding for the Rice Simulator Project to create data resources and simulation software aimed at driving plant research forward.

'This alone won't make for the second green revolution,' said Kenichi Higo, overall project leader and director of the molecular genetics department at the Japanese National Institute of Agrobiological Sciences (NIAS), a semi-governmental research institute. Nevertheless, access to a comprehensive bank of data and modelling software could allow breeders to develop varieties-whether genetically engineered or traditionally crossbredthat better match local climates. 'It's clear that the world will need wheat, corn, rice, or beans that can grow anywhere, in each locality, he said about the rationale for this undertaking.

The Japanese effort, in combination with the sequencing of the rice genome, is expected to have major economic and social implications. The production of rice is said to be the world's greatest economic activity. And while it feeds billions, serving as a staple for an estimated $50 \%$ of the world's population, its scientific importance is wholly disproportionate to its size. In fact, the rice genome is significant precisely because, at 430 million bases, it is the smallest of all the grains. Expectations are high that results from research on the rice genome will transfer quickly to the study of corn, wheat and other crops.

The groundwork for this latest Japanese project was laid by the eight-nation International Rice Genome Sequencing Project (IRGSP). Close behind the Beijing Institute and Syngenta, which sequenced the indica and japonica varieties, respectively, IRGSP is on schedule to complete a highly precise version-minimum 10-fold coverage with $99.99 \%$ accuracy, although allowing for some gaps-this year. With news reports of potential collaboration among the four major

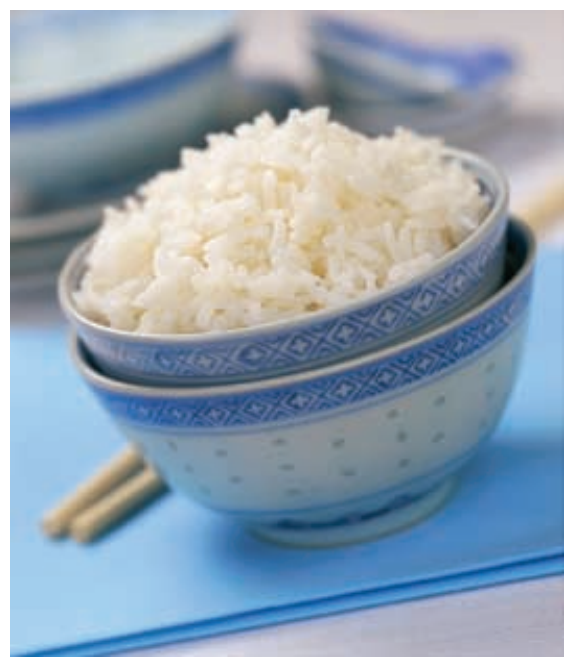

sequencing groups-Beijing, Syngenta, IRGSP and Monsanto, the latter having provided the early draft sequence data that IRGSP has drawn on-the complete sequence could be finished by 2003 . Commenting at the February IRGSP meeting in Tsukuba, Japan, Robin Buell, who heads the IRGSP effort at the Institute for Genomic Research (TIGR) in the USA, described the rice sequencing effort as a new public-private paradigm. Compared with the Human Genome Project, for

Access to a comprehensive bank of data and modelling software could allow breeders to develop varieties that better match local climates

example, the rice scientists have been more cooperative, she noted, sharing information in both directions.

This sequencing effort constitutes the majority of the Rice Genome Project (RGP), a Japan-wide network first organised under the auspices of the Ministry for Agriculture, Forestry and Fisheries (MAFF) in 1991. Reorganised in 1998, the RGP today involves over 220 laboratories across Japan; the majority are government institutions but $40 \%$ are housed in universities and private facilities.

With an eye to the future, several years ago researchers suggested coordinating the data produced under the RGP with developing technologies that would make the data accessible to a wide variety of plant specialists, including molecular biologists, plant physiologists and breeders. The result is the Rice Simulator Project, an experimental national scheme funded by MAFF to the tune of 1.2 billion Yen ( $€ 10.4$ million) per year to create a network of value-added databases where none currently exists. 'By combining life science and information science technologies, we aim to develop more specialised software and a system that can predict, in silico, gene functions in rice and other crops that will be used to conduct experiments to test hypotheses that could lead to improved crop varieties,' state the project administrators. Succinctly, the goals are to develop a database, software and a simulator to help develop new varieties.

Laboratories across the country are already amassing data from rice proteome analysis, full-length cDNA, microarray and knockout plants. The project will link the separate databases and accommodate continual data updates, giving as much conditional access as intellectual property concerns allow. These data will also form a network with publicly accessible resources such as the rice genome sequence, physical and genetic maps, and expression sequence tags.

The project received 34 applications from government facilities, universities and companies in its first year. Seventeen were approved in 2001 and given funding of up to 100 million Yen (€866 000) per year for 3 years. An additional five proposals were approved this February. The research is spread across public and private groups and is focused both locally and nationally with teams from Mitsubishi Space Software and Hitachi, and from universities, including Tokyo, Kyoto, Nagoya and Keio. All teams will be 


\section{analysis}

evaluated after the second year and those with little to show for their work could find their funding cut.

Conceptually, the 7-year project is divided into two segments. The aim of the first 3 years is to create a network of databases from sequence, expression and microarray data. The subsequent 4 years will be focused on creating in silico models of the plant at the level of single cells, tissues and the whole organism. Models for use in prediction studies will be developed in four areas: knockout simulation, stress response simulation, growth simulation and pollination, or breeding, simulation.

The participating scientists envisage the day when breeders can test the strengths and weaknesses of particular varieties of rice by crossbreeding them in silico. By accessing a database network housing sequence, expression and other genetic data, the specialist could hone in on a particular function and trace it all the way back to the gene level. The simulation software being developed under the project will also allow researchers to set up a model for viewing, for example, the effects of a drop in temperature on a plant at various stages of growth, such as sprouting, flowering or heading. This is the kind of information, they predict, that breeders will be demanding as they try to develop ever more niche-based varieties of rice.

In addition, the project aims to promote a number of secondary goals. Foremost is the drive to boost plant genomics in Japan, Higo said. Although the idea for the project originated with MAFF researchers, Higo hopes to spur more widespread interest in plant biology, in both academia and the corporate world. With a 'lift all boats' attitude, this meant giving priority during the selection process to proposals from groups that were expanding their expertise into this field for the first time.

As an extension of this thinking, evaluation of the proposals was geared towards encouraging collaboration between different research groups. But although this theme was stressed at project information sessions held early in the process, according to Higo, this has been a less successful element, one exception being the two grants awarded to Keio University's Masaru Tomita and Takaaki Nishioka of Kyoto University. Their two teams are joining forces, developing modelling software and supplying quantitative metabolic measurement data, respectively, to create a virtual plant, known as e-rice.

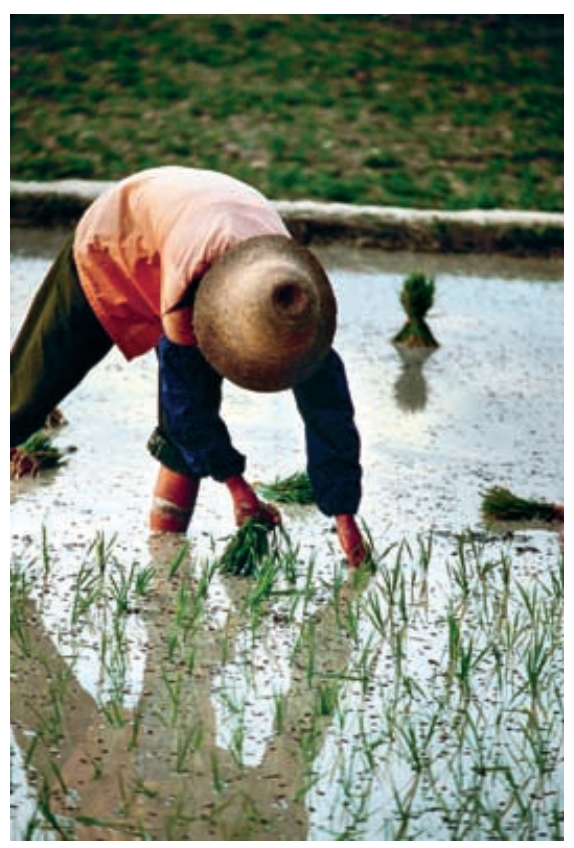

The approach of the Rice Simulator Project is to start with an outline and gradually fill in the details; as the amount of data grows, the system will need to expand. The 'bones' of this skeleton-first approach, according to Higo, will be the collection of data on major traits or from well-researched areas, for example the mechanism of flowering. The genetic basis for functions that affect taste, for example, are bound to come later.

\section{Robin Buell, from \\ TIGR, described the rice sequencing effort as a new public-private paradigm}

The two-part emphasis of the projectfirst on constructing databases, then on simulation-allows the project to allocate resources according to need. With steadily expanding amounts of data and the development of more sophisticated and detailed models, the computing needs of the project are continually growing. While there are now six central servers sustaining the project in addition to the computing capacity of each participating team, the computing resources that will be needed in the future remain unclear. No one knows how much power it takes to simulate a cell,' Higo said.
Constraints of time and money are also limiting the extent of the project's reach. As a result, cell and tissue simulation take priority over the modelling of protein changes and other aspects of molecular dynamics, since such detail would require massive amounts of computing power. 'In our case, we don't have plans at this time to elucidate all the genes in rice,' Higo said. 'We just want to construct virtual life.'

Officials will also have to decide about the accessibility of the project. As planned, it would both take advantage of public resources worldwide and serve international researchers. But in practice, it is clear that the priority is on domestic research. This is a problem, argues Kiyotaka Okada, a plant biologist from Kyoto University. II should be open for the researchers in other countries and should include a wide collaboration with projects of other countries,' he said. However, he concedes that there might be problems, 'because research on rice is closely related to the national food production policy.'

The Japanese approach contrasts markedly with the strategy of the US-led 2010 Project, which focuses on Arabidopsis. In fact, its predecessor, the Arabidopsis Genome Research Project, and Japan's Rice Genome Project have parallel histories and actually overlap with the start of the international sequencing consortium effort in 1998. At a 1999 hearing on plant genome science, held by a US House of Representatives subcommittee overseeing basic research, Mary Clutter, then assistant director for biological research at the National Science Foundation, outlined the 10-year goals for Arabidopsis formulated by scientists in the plant biology community. Their aims, she said, were 'to exploit the revolution in plant genomics by understanding the function of all Arabidopsis genes (and, by extension, the genes of all flowering plants) within their cellular, organismal and evolutionary context; and to create an information structure to coordinate, integrate, analyse and make accessible this knowledge.' Thus, the 2010 Project sets out to clarify the function of all 25000 Arabidopsis genes and create a 'clickable' plant model within the first decade of the new millennium. Those discussions had a big impact on Japanese researchers, noted Okada, who represented Japan at the 2010 Project meetings. The 


\section{analysis}

Japanese Society of Plant Physiologists even reacted with a 10-year plan for domestic researchers.

Projects such as these put the emphasis on the future of agriculture and the food supply, as they allow researchers not only to adapt crops to particular conditions but also to improve their nutritional value. The development of Golden Rice, the orange-coloured rice that produces a surplus of Vitamin A, has already drawn attention to the field of nutritional genomics and the ways in which food can be used to improve health. As Higo sees it, the Rice Simulator Project will create the technological engine to drive future development of crop varieties. Traditionally, the emphasis has been on increasing yield, through which the successes of the 'green revolution' were built. Short-term applications for Japan-based breeders, he predicts, will be focused on better tailoring plants to regional climate conditions-from cold Hokkaido in the north to the subtropical paddy fields of Okinawa. Such research could then be expanded to fit an international context.
Researchers also have their eyes on 'designer' rice, special varieties for which consumers can be expected to pay a premium. In addition to good taste, this

\section{Perhaps the greatest importance of rice lies in what it can tell scientists about other plants}

would include varieties with medicinal properties: rice that lowers blood pressure, or supplies enough iron to stave off anaemia.

But perhaps the greatest importance of rice lies in what it can tell scientists about other plants, and not just grains. Rice, because of its size, is simply the easiest cereal grain with which to work; researchers hold high expectations that it will help to demystify the functions of homologous regions in wheat and corn. In comparison, the corn genome is thought to have 3 billion base pairs, whereas wheat has 16 billion. Conversely, comparisons of the monocotyledon rice with the dicotyledonous Arabidopsis will help to iden- tify common denominators in plants. 'We know the same set of genes are working in the very basic developmental processes,' Okada said, but 'it is important that we will know that not all results from Arabidopsis are common in plants.'

Using rice genome research to clarify the details of common plant functionsincluding those not consumed for food-could further expand its effects. Take the example of Chinese medicine, Higo said, or other plant-derived drugs. 'There are lots of plants that are rare and therefore expensive,' he notes. 'But find those genes and they can be made in quantity, and sold cheaply.' Clearly, rice is seen only as the first step. 'We are not studying rice in order to develop new rice varieties,' Higo says. 'Rather, as we work on rice we are thinking about the unlimited possibilities of plant biology.'

\section{Sara B. Harris}

DOI: 10.1093/embo-reports/kvf125 